\title{
Computation of Edge Resolvability of Benzenoid Tripod Structure
}

\author{
Ali Ahmad $\mathbb{D}$, ${ }^{1}$ Sadia Husain, ${ }^{1}$ Muhammad Azeem $\left(\mathbb{D},{ }^{2}\right.$ Kashif Elahi, ${ }^{3}$ and M. K. Siddiqui ${ }^{4}{ }^{4}$ \\ ${ }^{1}$ College of Computer Science \& Information Technology, Jazan University, Jazan, Saudi Arabia \\ ${ }^{2}$ Department of Mathematics, Riphah Institute of Computing and Applied Sciences, Riphah International University, \\ Lahore, Pakistan \\ ${ }^{3}$ Deanship of E-Learning and Information Technology, Jazan University, Jazan, Saudi Arabia \\ ${ }^{4}$ Department of Mathematics, COMSATS University Islamabad, Lahore Campus, Lahore 54000, Pakistan
}

Correspondence should be addressed to Ali Ahmad; ahmadsms@gmail.com

Received 14 August 2021; Accepted 9 October 2021; Published 21 October 2021

Academic Editor: Gaetano Luciano

Copyright (c) 2021 Ali Ahmad et al. This is an open access article distributed under the Creative Commons Attribution License, which permits unrestricted use, distribution, and reproduction in any medium, provided the original work is properly cited.

In chemistry, graphs are commonly used to show the structure of chemical compounds, with nodes and edges representing the atom and bond types, respectively. Edge resolving set $\lambda_{e}$ is an ordered subset of nodes of a graph $C$, in which each edge of $C$ is distinctively determined by its distance vector to the nodes in $\lambda$. The cardinality of a minimum edge resolving set is called the edge metric dimension of $C$. An edge resolving set $L_{e, f}$ of $C$ is fault-tolerant if $\lambda_{e, f} \backslash b$ is also an edge resolving set, for every $b$ in $\lambda_{e, f}$. Resolving set allows obtaining a unique representation for chemical structures. In particular, they were used in pharmaceutical research for discovering patterns common to a variety of drugs. In this paper, we determine the exact edge metric and faulttolerant edge metric dimension of benzenoid tripod structure and proved that both parameters are constant.

\section{Introduction}

Mathematical chemistry has recently presented a wide range of approaches to understanding the chemical structures that underpin existing chemical theories and developing and exploring new mathematical models of chemical phenomena and applying mathematical concepts and processes to chemistry. Only a few scientists have been convinced to exploit linkages between mathematics and chemistry and the possibility of using arithmetic to deduce known and anticipate new chemical characteristics, throughout the history of science. In many areas of physical chemistry, such as thermodynamics and compound energy, extensive use of mathematical approaches is commonplace. After physicists revealed in the first few years of the twentieth century that the key features of chemical compounds can be predicted using quantum theory approaches, a significant need for mathematics in chemistry arose. The main driving force that drove the mathematics and its concepts into chemistry laboratories was the realization that chemistry cannot be comprehended without knowledge of quantum physics, including its complicated mathematical instruments. For the different study of mathematical chemistry in terms of graph theory, we suggest some literature here [1].

Chemical graph theory is a branch of mathematical science that is used to characterise the structural properties of molecules, processes, crystals, polymers, clusters, and other objects. The vertex of a chemical graph theory might be an electron, an atom, a molecule, a collection of atoms, intermediates, orbitals, and many other things. Intermolecular bonding, bonded and nonbonded connections, basic reactions, and other forces such as van der Waals forces, Keesom forces, and Debye forces can all be used to illustrate the relationships between vertices of a structure.

The general convex polytope structures are discussed in $[2,3]$, in which authors consider the problem of edge metric resolvability. In the reply to aroused question from seminal work of edge resolvability, Raza and Bataineh [4] answered some questions and provided interesting results as well as analysis between vertex and edge resolvability. The detailed discussion of identifying edges and vertices of general graph is studied in [5]. Necklace graph's edge resolvability is discussed in [6]. Polycyclic aromatic hydrocarbons in terms of edge and fault-tolerant edge resolvability are deeply 
investigated in [7]. The generalized version of edge resolvability is introduced in [8]. Few efficient techniques of finding edge resolving set are found in [9]. The graph having larger edge resolvability rather than vertex resolvability is generally studied in [10]. The generalized Peterson graph's edge resolvability is found in [11]. The k-multiwheel graph point of discussion with its edge resolvability is found in [12].

Slater [13] presented the idea of resolving sets, which was later discussed by Harary and Melter [14]. Metric generators, as detailed in $[15,16]$, allow for alternative representations of chemical substances. Precisely, they were used in pharmaceutical research for determining patterns similar to a variety of drugs [17]. Metric dimension has various other applications, such as robot navigation [18], weighing problems [19], computer networks [20], combinatorial optimization [21], image processing, facility location problems, and sonar and coastguard loran [13]; for further detail, see [22, 23]. Due to its variety of applications, the concept of metric dimension is widely used to solve many difficult problems. Hussain et al. [24], Krishnan et al. [25], and Siddiqui et al. [26] have computed the resolvability parameter for alphaboron nanotube, certain crystal structures, and certain nanotube lattices, respectively. For the NP-harness of these topics, for example, and for the metric dimension [27, 28] and application along with its NP-hardness, see [29]. Rather than separating two unique vertices of a graph based on a subset of vertices, two edges might be distinguished based on the same subset of vertices. Kelenc et al. [30] created a new parameter called the edge metric dimension to represent this idea. They employed graph metric to identify each pair of edges based on the graph's distance from a selection of vertices.

The following are basic preliminaries for the concepts studied here.

Definition 1 (see [31]). Assume that $C$ is an associated graph of chemical structure/network, whose vertex/node set are denoted by symbol $N(C)$ or simply $N$, while $B(C)$ or $B$ is the edge/bond set, and the shortest distance between two bonds $b_{1}, b_{2} \in N(C)$ is denoted by $S_{b_{1}, b_{2}}$ and calculated by counting the number of bonds while moving through the $b_{1}-b_{2}$ path.
Definition 2 (see [32]). The distance between an edge $e=$ $b_{1} b_{2} \in B(C)$ and a node $b \in N(C)$ is counted by the relation $S e, b=\min \left\{S b_{1}, b, S b_{2}, b\right\}$. Assuming a subset of selected nodes $\lambda_{e}$, if the position $\mathfrak{p}\left(e \mid \lambda_{e}\right)$ of each edge $e$ is unique of a graph, then $\lambda_{e}$ is called as edge metric resolving set and $\operatorname{dim}_{e}(C)$ is the minimum count of members of $\lambda_{e}$, called as edge metric dimension.

Definition 3. Assuming that any of the member of edge metric resolving set $\lambda_{e}$ is not working or any of the node from $\kappa$ members is spoiled, then one cannot get the unique position of the entire edge set. To tackle this issue, the definition is known as fault-tolerant edge resolving set which is dealt with by eliminating any of the member from $\lambda_{e}$ and still obtains the unique position of the entire edge set of a graph, symbolized as $\lambda_{e, f}$, and the minimum members in the set denoted as $\operatorname{dim}_{e, f}(C)$ and named as fault-tolerant edge metric dimension.

Theorem 1 (see [30]). If $\operatorname{dim}_{e}(C)$ is the edge metric dimension, then $\operatorname{dim}_{e}(C)=1$, iff $C$ is a path $P_{n}$.

\section{Construction of Tripod Structure}

Because it is significant in theoretical chemistry, benzenoid systems are natural graph representations of benzenoid hydrocarbons. It is a well-known fact that hydrocarbons generated from benzenoids are important and beneficial in the chemical, food, and environmental industries, according to [33]. The authors in [34] describe the benzenoid system we mentioned above in our work. Polynomial types were discussed in relation to various catacondensed and pericondensed benzenoid structures. This is a pericondensed benzenoid tripod construction. It has $4\left(\delta_{1}+\delta_{2}+\delta_{3}\right)-8$ nodes and $5\left(\delta_{1}+\delta_{2}+\delta_{3}\right)-11$ bonds, with all the running parameters $\delta_{1}, \delta_{2}, \delta_{3} \geq 2$. Furthermore, Jamil et al. [35] provide a comprehensive topological investigation of benzenoid structures, and metric-based study of benzenoid networks is available in [36]. The node and bond or vertex and edge set for the benzenoid structure $T\left(\delta_{1}, \delta_{2}, \delta_{3}\right)$ is shown as follows. In our primary results, we apply the labelling of nodes and edges specified in Figure 1.

$$
\begin{aligned}
N\left(T\left(\delta_{1}, \delta_{2}, \delta_{3}\right)\right)= & \left\{a_{\kappa}: 1 \leq \kappa \leq 2 \delta_{3}\right\} \cup\left\{b_{\kappa}: 1 \leq \kappa \leq 2 \delta_{1}\right\} \cup\left\{c_{\kappa}, c_{\kappa}^{\prime}: 1 \leq \kappa \leq 2 \delta_{2}-1\right\} \\
& \cup\left\{a_{\kappa}^{\prime}: 1 \leq \kappa \leq 2 \delta_{3}-3\right\} \cup\left\{b_{\kappa}^{\prime}: 1 \leq \kappa \leq 2 \delta_{1}-3\right\}, \\
B\left(T\left(\delta_{1}, \delta_{2}, \delta_{3}\right)\right)= & \left\{a_{\kappa} a_{\kappa+1}: 1 \leq \kappa \leq 2 \delta_{3}-1\right\} \cup\left\{b_{\kappa} b_{\kappa+1}: 1 \leq \kappa \leq 2 \delta_{1}-1\right\} \\
& \cup\left\{c_{\kappa} c_{\kappa+1}, c_{\kappa}^{\prime} c_{\kappa+1}^{\prime}: 1 \leq \kappa \leq 2 \delta_{2}-2\right\} \cup\left\{a_{\kappa}^{\prime} a_{\kappa+1}^{\prime}: 1 \leq \kappa \leq 2 \delta_{3}-4\right\} \\
& \cup\left\{b_{\kappa}^{\prime} b_{\kappa+1}^{\prime}: 1 \leq \kappa \leq 2 \delta_{1}-4\right\} \cup\left\{a_{\kappa} a_{\kappa}^{\prime}: 1 \leq \kappa(\text { odd }) \leq 2 \delta_{3}-3\right\} \\
& \cup\left\{b_{\kappa+3} b_{\kappa}^{\prime}: 1 \leq \kappa(\text { odd }) \leq 2 \delta_{1}-3\right\} \cup\left\{c_{\kappa} c_{\kappa}^{\prime}: 1 \leq \kappa(\text { odd }) \leq 2 \delta_{2}-1\right\} \\
& \cup\left\{a_{2 \delta_{3}} b_{1}, a_{2 \delta_{3}-1} c_{1}^{\prime}, b_{2} c_{1}, a_{2 \delta_{3}-3} c_{2} c_{2}^{\prime}, b_{1}^{\prime} c_{2}\right\} .
\end{aligned}
$$




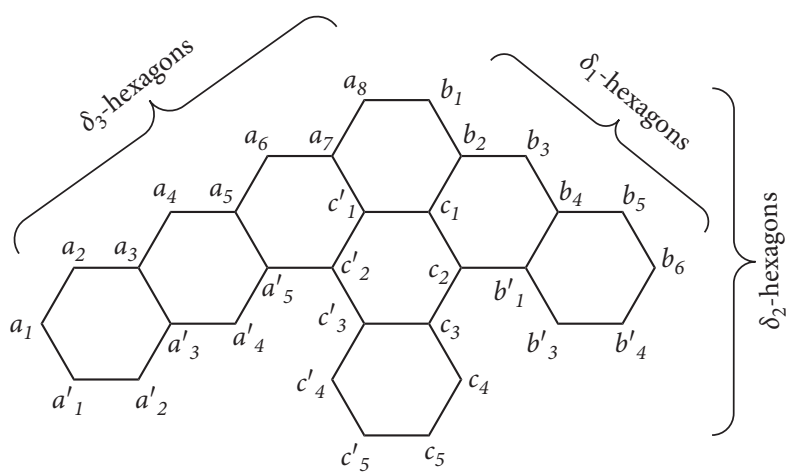

Figure 1: Benzenoid tripod with $\left\{\delta_{1}, \delta_{2}, \delta_{3}\right\}=\{3,3,4\}$.

Lemma 1. If $T\left(\delta_{1}, \delta_{2}, \delta_{3}\right)$ is a graph of benzenoid tripod with $\delta_{1}, \delta_{2}, \delta_{3} \geq 2$, then the minimum members in its edge resolving set are two.

Proof. The total number of nodes in the corresponding graph of benzenoid tripod with $\delta_{1}, \delta_{2}, \delta_{3} \geq 2$ is $4\left(\delta_{1}+\delta_{2}+\right.$ $\left.\delta_{3}\right)-8$ and to check the possibilities of edge resolving set with cardinality two is $C\left(4\left(\delta_{1}+\delta_{2}+\delta_{3}\right)-8,2\right)=$ $\left(\left(4\left(\delta_{1}+\delta_{2}+\delta_{3}\right)-8\right) ! /\left(2 \times\left(4\left(\delta_{1}+\delta_{2}+\delta_{3}\right)-10\right) !\right)\right)$. Here, we are checking with cardinality two because by Theorem 1 , the edge resolving set with cardinality one is reserved for path graph only. Now due to the NP-hardness of choosing edge resolving set, we cannot find the exact number of edge resolving sets for a graph, but from $\left(\left(4\left(\delta_{1}+\delta_{2}+\delta_{3}\right)-8\right) ! /\left(2 \times\left(4\left(\delta_{1}+\delta_{2}+\delta_{3}\right)-10\right) !\right)\right)$-possibilities, we choose a subset $\lambda_{e}$ and defined as $\lambda_{e}=\left\{a_{1}, b_{1}\right\}$. Now to prove this claim that $\lambda_{e}$ is actually one of the candidates for the edge resolving set of benzenoid tripod graph or $T\left(\delta_{1}, \delta_{2}, \delta_{3}\right)$, we will follow Definition 2. To fulfill the requirements of definition, we will check the unique positions or locations of each edge and the methodology is defined above in Definition 2.

Positions $\mathfrak{p}\left(a_{\kappa} a_{\kappa+1} \mid \lambda_{e}\right)$ with respect to $\lambda_{e}$, for the edges $a_{\kappa} a_{\kappa+1}$ with $\kappa=1,2, \ldots, 2 \delta_{3}-1$, are given as

$$
\mathfrak{p}\left(a_{\kappa} a_{\kappa+1} \mid \lambda_{e}\right)=\left(\kappa-1,2 \delta_{3}-\kappa\right) .
$$

Positions $\mathfrak{p}\left(b_{\kappa} b_{\kappa+1} \mid \lambda_{e}\right)$ with respect to $\lambda_{e}$, for the edges $b_{\kappa} b_{\kappa+1}$ with $\kappa=1,2, \ldots, 2 \delta_{1}-1$, are given as

$$
\mathfrak{p}\left(b_{\kappa} b_{\kappa+1} \mid \lambda_{e}\right)=\left(2 \delta_{3}+\kappa-1, \kappa-1\right)
$$

Positions $\mathfrak{p}\left(c_{\kappa} c_{\kappa+1} \mid \lambda_{e}\right)$ with respect to $\lambda_{e}$, for the edges $c_{\kappa} c_{\kappa+1}$ with $\kappa=1,2, \ldots, 2 \delta_{2}-2$, are given as

$$
\mathfrak{p}\left(c_{\kappa} c_{\kappa+1} \mid \lambda_{e}\right)= \begin{cases}\left(2 \delta_{3}, \kappa+1\right), & \text { if } \kappa=1,2, \\ \left(2 \delta_{3}+\kappa-3, \kappa+1\right), & \text { if } \kappa=3,4, \ldots, 2 \delta_{2}-1 .\end{cases}
$$

Positions $\mathfrak{p}\left(a_{\kappa}^{\prime} a_{\kappa+1}^{\prime} \mid \lambda_{e}\right)$ with respect to $\lambda_{e}$, for the edges $a_{\kappa}^{\prime} a_{\kappa+1}^{\prime}$ with $\kappa=1,2, \ldots, 2 \delta_{3}-4$, are given as

$$
\mathfrak{p}\left(a_{\kappa}^{\prime} a_{\kappa+1}^{\prime} \mid \lambda_{e}\right)=\left(\kappa, 2 \delta_{3}+1-\kappa\right) .
$$

Positions $\mathfrak{p}\left(b_{\kappa}^{\prime} b_{\kappa+1}^{\prime} \mid \lambda_{e}\right)$ with respect to $\lambda_{e}$, for the edges $b_{\kappa}^{\prime} b_{\kappa+1}{ }^{\prime}$ with $\kappa=1,2, \ldots, 2 \delta_{1}-4$, are given as

$$
\mathfrak{p}\left(b_{\kappa}^{\prime} b_{\kappa+1}^{\prime} \mid \lambda_{e}\right)=\left(2 \delta_{3}+\kappa+1, \kappa+3\right)
$$

Positions $\mathfrak{p}\left(c_{\kappa}^{\prime} c_{\kappa+1}^{\prime} \mid \lambda_{e}\right)$ with respect to $\lambda_{e}$, for the edges $c_{\kappa}^{\prime} c_{\kappa+1}^{\prime}$ with $\kappa=1,2, \ldots, 2 \delta_{2}-2$, are given as

$$
\mathfrak{p}\left(c_{\kappa}^{\prime} c_{\kappa+1}^{\prime} \mid \lambda_{e}\right)= \begin{cases}\left(2\left(\delta_{3}-1\right), \kappa+2\right), & \text { if } \kappa=1, \\ \left(2\left(\delta_{3}-2\right)+\kappa, \kappa+2\right), & \text { if } \kappa=2,3, \ldots, 2 \delta_{2}-1 .\end{cases}
$$

Positions $\mathfrak{p}\left(a_{\kappa} a_{\kappa}^{\prime} \mid \lambda_{e}\right)$ with respect to $\lambda_{e}$, for the edges $a_{\kappa} a_{\kappa}^{\prime}$ with $\kappa=1,3, \ldots, 2 \delta_{3}-3$, are given as

$$
\mathfrak{p}\left(a_{\kappa} a_{\kappa}^{\prime} \mid \lambda_{e}\right)=\left(\kappa-1,2 \delta_{3}-\kappa+1\right) .
$$

Positions $\mathfrak{p}\left(b_{\kappa+3} b_{\kappa}^{\prime} \mid \lambda_{e}\right)$ with respect to $\lambda_{e}$, for the edges $b_{\kappa+3} b_{\kappa}^{\prime}$ with $\kappa=1,3, \ldots, 2 \delta_{1}-3$, are given as

$$
\mathfrak{p}\left(b_{\kappa+3} b_{\kappa}^{\prime} \mid \lambda_{e}\right)=\left(2 \delta_{3}+\kappa+1, \kappa+2\right) .
$$

Positions $\mathfrak{p}\left(c_{\kappa} a_{\kappa}^{\prime} \mid \lambda_{e}\right)$ with respect to $\lambda_{e}$, for the edges $c_{\kappa} c_{\kappa}^{\prime}$ with $\kappa=1,3, \ldots, 2 m l-3$, are given as

$$
\mathfrak{p}\left(c_{\kappa} c_{\kappa}^{\prime} \mid \lambda_{e}\right)= \begin{cases}\left(2 \delta_{3}-1, \kappa+1\right), & \text { if } \kappa=1, \\ \left(2\left(\delta_{3}-2\right)+\kappa, \kappa+1\right), & \text { if } \kappa=2,3, \ldots, 2 \delta_{2}-1 .\end{cases}
$$

Positions of the joint edges are given as

$$
\begin{aligned}
\mathfrak{p}\left(a_{2 \delta_{3}} b_{1} \mid \lambda_{e}\right) & =\left(2 \delta_{3}-1,0\right), \\
\mathfrak{p}\left(a_{2 \delta_{3}} c_{1}^{\prime} \mid \lambda_{e}\right) & =\left(2 \delta_{3}-2,2\right), \\
\mathfrak{p}\left(b_{2} c_{1} \mid \lambda_{e}\right) & =\left(2 \delta_{3}, 1\right), \\
\mathfrak{p}\left(a_{2 \delta_{3}-3}{ }^{\prime} c_{2}^{\prime} \mid \lambda_{e}\right) & =\left(2 \delta_{3}-3,4\right), \\
\mathfrak{p}\left(b_{1}^{\prime} c_{2} \mid \lambda_{e}\right) & =\left(2 \delta_{3}+1,3\right) .
\end{aligned}
$$

The given positions $\mathfrak{p}\left(. \mid \lambda_{e}\right)$ of all $5\left(\delta_{1}+\delta_{2}+\delta_{3}\right)-11$-bonds of $T\left(\delta_{1}, \delta_{2}, \delta_{3}\right)$ graph of benzenoid tripod with $\delta_{1}, \delta_{2}, \delta_{3} \geq 2$, with respect to $\lambda_{e}$, are unique and no two bonds have the same position $\mathfrak{p}$. So we can conclude that we resolve the bonds of $T\left(\delta_{1}, \delta_{2}, \delta_{3}\right)$ with two nodes. It is implied that the minimum members in the edge resolving set of $T\left(\delta_{1}, \delta_{2}, \delta_{3}\right)$ are two.

Remark 1. If $T\left(\delta_{1}, \delta_{2}, \delta_{3}\right)$ is a graph of benzenoid tripod with $\delta_{1}, \delta_{2}, \delta_{3} \geq 2$, then

$$
\operatorname{dim}_{e}\left(T\left(\delta_{1}, \delta_{2}, \delta_{3}\right)\right)=2 .
$$

Proof. From the definition of edge metric dimension, the concept is solemnly based on the selected subset $\left(\lambda_{e}\right)$ chosen in such a way that the entire edge set has unique position with respect to the selected nodes or subset. In Lemma 1, we already discussed the possibility of selected subset (edge resolving set) and, according to the definition, its minimum possible cardinality. In that lemma, we choose $\lambda_{e}=\left\{a_{1}, b_{1}\right\}$ as an edge resolving set for the graph of benzenoid tripod or $T\left(\delta_{1}, \delta_{2}, \delta_{3}\right)$ for all the possible combinatorial values of $\delta_{1}, \delta_{2}, \delta_{3} \geq 2$. We also proved in such lemma that $\left|\lambda_{e}\right|=2$ is the least possible cardinality of edge resolving set for the 
benzenoid tripod $T\left(\delta_{1}, \delta_{2}, \delta_{3}\right)$. It is enough for the proof of what we claim in the statement that edge metric dimension of benzenoid tripod is two, which completes the proof.

Lemma 2. If $T\left(\delta_{1}, \delta_{2}, \delta_{3}\right)$ is a graph of benzenoid tripod with $\delta_{1}, \delta_{2}, \delta_{3} \geq 2$, then the minimum members in its faulttolerant edge resolving set are four.

Proof. The total number of nodes in the corresponding graph of benzenoid tripod with $\delta_{1}, \delta_{2}, \delta_{3} \geq 2$, are $4\left(\delta_{1}+\delta_{2}+\right.$ $\left.\delta_{3}\right)-8$ and to check the possibilities of fault-tolerant edge resolving set with cardinality four are $C\left(4\left(\delta_{1}+\delta_{2}+\delta_{3}\right)-\right.$ $8,4)=\left(\left(4\left(\delta_{1}+\delta_{2}+\delta_{3}\right)-8\right) ! /\left(2 \times\left(4\left(\delta_{1}+\delta_{2}+\delta_{3}\right)-12\right) !\right)\right)$. Here we are checking with cardinality four, and later, we will also check cardinality three. Now due to the NP-hardness of choosing fault-tolerant edge resolving set, we cannot find the exact number of fault-tolerant edge resolving sets for a graph, but from $\left(\left(4\left(\delta_{1}+\delta_{2}+\delta_{3}\right)-8\right) ! /\left(2 \times\left(4\left(\delta_{1}+\delta_{2}+\right.\right.\right.\right.$ $\left.\left.\delta_{3}\right)-12\right)$ !))-possibilities, we choose a subset $\lambda_{e, f}$ and defined as $\lambda_{e, f}=\left\{a_{1}, b_{1}, a_{2 \delta_{3}}, b_{2 \delta_{1}}\right\}$. Now to prove this claim that $\lambda_{e, f}$ is actually one of the candidates for the fault-tolerant edge resolving set of benzenoid tripod graph or $T\left(\delta_{1}, \delta_{2}, \delta_{3}\right)$, we will follow Definition 3. To fulfill the requirements of definition, we will check the unique positions or locations of each node and the methodology is defined above in Definition 3.

Positions $\mathfrak{p}\left(a_{\kappa} a_{\kappa+1} \mid \lambda_{e, f}\right)$ with respect to $\lambda_{e, f}$, for the edges $a_{\kappa} a_{\kappa+1}$ with $\kappa=1,2, \ldots, 2 \delta_{3}-1$, are given as

$$
\mathfrak{p}\left(a_{\kappa} a_{\kappa+1} \mid \lambda_{e, f}\right)= \begin{cases}\left(\kappa-1,2 \delta_{3}-\kappa, 2 \delta_{3}-1-\kappa, 2\left(\delta_{3}+\delta_{1}\right)-1-\kappa\right), & \text { if } \kappa=1,2, \ldots, 2 \delta_{3}-2, \\ \left(\kappa-1,2 \delta_{3}-\kappa, 2 \delta_{3}-1-\kappa, 2 \delta_{3}\right), & \text { if } \kappa=2 \delta_{3}-1 .\end{cases}
$$

Positions $\mathfrak{p}\left(b_{\kappa} b_{\kappa+1} \mid \lambda_{e, f}\right)$ with respect to $\lambda_{e, f}$, for the edges $b_{\kappa} b_{\kappa+1}$ with $\kappa=1,2, \ldots, 2 \delta_{1}-1$, are given as

$$
\mathfrak{p}\left(b_{\kappa} b_{\kappa+1} \mid \lambda_{e, f}\right)=\left(2 \delta_{3}+\kappa-1, \kappa-1, \kappa, 2 \delta_{1}-1-\kappa\right) .
$$

Positions $\mathfrak{p}\left(c_{\kappa} c_{\kappa+1} \mid \lambda_{e, f}\right)$ with respect to $\lambda_{e, f}$, for the edges $c_{\kappa} c_{\kappa+1}$ with $\kappa=1,2, \ldots, 2 \delta_{2}-2$, are given as

$$
\mathfrak{p}\left(c_{\kappa} c_{\kappa+1} \mid \lambda_{e, f}\right)= \begin{cases}\left(2 \delta_{3}, \kappa+1, \kappa+2,2 \delta_{1}-2\right), & \text { if } \kappa=1, \\ \left(2 \delta_{3}, \kappa+1, \kappa+2,2\left(\delta_{1}-2\right)+\kappa\right), & \text { if } \kappa=2, \\ \left(2 \delta_{3}+\kappa-3, \kappa+1, \kappa+2,2\left(\delta_{1}-2\right)+\kappa\right), & \text { if } \kappa=3,4, \ldots, 2 \delta_{2}-1\end{cases}
$$

Positions $\mathfrak{p}\left(a_{\kappa}^{\prime} a_{\kappa+1}^{\prime} \mid \lambda_{e, f}\right)$ with respect to $\lambda_{e, f}$, for the edges $a_{\kappa}^{\prime} a_{\kappa+1}^{\prime}$ with $\kappa=1,2, \ldots, 2 \delta_{3}-4$, are given as

$$
\mathfrak{p}\left(a_{\kappa}^{\prime} a_{\kappa+1}^{\prime} \mid \lambda_{e, f}\right)=\left(\kappa, 2 \delta_{3}+1-\kappa, 2 \delta_{3}-\kappa, 2\left(\delta_{3}+\delta_{1}-1\right)-\kappa\right) .
$$

$$
\mathfrak{p}\left(b_{\kappa}^{\prime} b_{\kappa+1}^{\prime} \mid \lambda_{e, f}\right)=\left(2 \delta_{3}+\kappa+1, \kappa+3, \kappa+4,2 \delta_{1}-3-\kappa\right) .
$$

Positions $\mathfrak{p}\left(c_{\kappa}^{\prime} c_{\kappa+1}^{\prime} \mid \lambda_{e, f}\right)$ with respect to $\lambda_{e, f}$, for the edges $c_{\kappa}^{\prime} c_{\kappa+1}^{\prime}$ with $\kappa=1,2, \ldots, 2 \delta_{2}-2$, are given as

Positions $\mathfrak{p}\left(b_{\kappa}^{\prime} b_{\kappa+1}{ }^{\prime} \mid \lambda_{e, f}\right)$ with respect to $\lambda_{e, f}$, for the edges $b_{\kappa}^{\prime} b_{\kappa+1}^{\prime}$ with $\kappa=1,2, \ldots, 2 \delta_{1}-4$, are given as

$$
\mathfrak{p}\left(c_{\kappa}^{\prime} c_{\kappa+1}^{\prime} \mid \lambda_{e, f}\right)= \begin{cases}\left(2\left(\delta_{3}-1\right), \kappa+2, \kappa+1,2 \delta_{1}\right), & \text { if } \kappa=1, \\ \left(2\left(\delta_{3}-1\right), \kappa+2, \kappa+1,2 \delta_{1}\right), & \text { if } \kappa=2, \\ \left(2\left(\delta_{3}-2\right)+\kappa, \kappa+2, \kappa+1,2 \delta_{1}-3+\kappa\right), & \text { if } \kappa=3,4, \ldots, 2 \delta_{2}-1\end{cases}
$$

Positions $\mathfrak{p}\left(a_{\kappa} a_{\kappa}^{\prime} \mid \lambda_{e, f}\right)$ with respect to $\lambda_{e, f}$, for the edges $a_{\kappa} a_{\kappa}^{\prime}$ with $\kappa=1,3, \ldots, 2 \delta_{3}-3$, are given as:

$$
\mathfrak{p}\left(a_{\kappa} a_{\kappa}^{\prime} \mid \lambda_{e, f}\right)=\left(\kappa-1,2 \delta_{3}-\kappa+1,2 \delta_{3}-\kappa, 2\left(\delta_{3}+\delta_{1}\right)-1-\kappa\right) .
$$

Positions $\mathfrak{p}\left(b_{\kappa+3} b_{\kappa}^{\prime} \mid \lambda_{e, f}\right)$ with respect to $\lambda_{e, f}$, for the edges $b_{\kappa+3} b_{\kappa}^{\prime}$ with $\kappa=1,3, \ldots, 2 \delta_{1}-3$, are given as

$$
\mathfrak{p}\left(b_{\kappa+3} b_{\kappa}^{\prime} \mid \lambda_{e, f}\right)=\left(2 \delta_{3}+\kappa+1, \kappa+2, \kappa+3,2 \delta_{1}-3-\kappa\right) .
$$


Positions $\mathfrak{p}\left(c_{\kappa} a_{\kappa}^{\prime} \mid \lambda_{e, f}\right)$ with respect to $\lambda_{e, f}$, for the edges $c_{\kappa} c_{\kappa}^{\prime}$ with $\kappa=1,3, \ldots, 2 m l-3$, are given as

$$
\mathfrak{p}\left(c_{\kappa} c_{\kappa}^{\prime} \mid \lambda_{e, f}\right)= \begin{cases}\left(2 \delta_{3}-1, \kappa+1, \kappa+1,2 \delta_{1}-1\right), & \text { if } \kappa=1, \\ \left(2\left(\delta_{3}-2\right)+\kappa, \kappa+1, \kappa+1,2\left(\delta_{1}-2\right)+\kappa\right), & \text { if } \kappa=2,3, \ldots, 2 \delta_{2}-1\end{cases}
$$

Positions of the joint edges with respect to $\lambda_{e, f}$ are given as

$$
\begin{aligned}
\mathfrak{p}\left(a_{2 \delta_{3}} b_{1} \mid \lambda_{e, f}\right) & =\left(2 \delta_{3}-1,0,0,2 \delta_{1}-1\right), \\
\mathfrak{p}\left(a_{2 \delta_{3}} c_{1}^{\prime} \mid \lambda_{e, f}\right) & =\left(2 \delta_{3}-2,2,1,2 \delta_{1}\right), \\
\mathfrak{p}\left(b_{2} c_{1} \mid \lambda_{e, f}\right) & =\left(2 \delta_{3}, 1,2,2 \delta_{1}-2\right), \\
\mathfrak{p}\left(a_{2 \delta_{3}-3}^{\prime} c_{2}^{\prime} \mid \lambda_{e, f}\right) & =\left(2 \delta_{3}-3,4,3,2 \delta_{1}+1\right), \\
\mathfrak{p}\left(b_{1}^{\prime} c_{2} \mid \lambda_{e, f}\right) & =\left(2 \delta_{3}+1,3,4,2 \delta_{1}-3\right) .
\end{aligned}
$$

On the behalf of given fact for the fulfillment of definition of fault-tolerant edge resolving set, we can say that $\lambda_{e, f}$ with cardinality four is possible, but when it comes to optimized value of $\left|\lambda_{e, f}\right|$, we still need to investigate about the minimum value of $\left|\lambda_{e, f}\right|$. Following are some possible cases to check that whether $\left|\lambda_{e, f}\right|=3$ is possible or not. However, we find the fault-tolerant edge resolving set with the help of algorithm satisfying that $\left|\lambda_{e, f}\right| \neq 3$, but for the proving purpose we build some general cases and try to conclude that only $\left|\lambda_{e, f}\right|>3$ is possible.

Case 1: assume that $\lambda_{e, f}{ }^{\prime} \subset\left\{a_{\kappa}: \kappa=1,2, \ldots, 2 \delta_{3}\right\}$, with a condition according to our requirement of theorem that $\left|\lambda_{e, f}{ }^{\prime}\right|=3$, and removal of any vertex from $\lambda_{e, f}{ }^{\prime}$ to fulfill the definition. The result is implied in the same edge's position and contradicts our assumption with the fact that $p\left(a_{r} a_{r+1} \mid \lambda_{e, f}^{\prime}\right)=p\left(a_{s} a_{s}^{\prime} \mid \lambda_{e, f}^{\prime}\right)$, where $1 \leq r \leq 2 \delta_{3}-1$ and $1 \leq s($ odd $) \leq 2 \delta_{3}-3$.

Case 2: assume that $\lambda_{e, f} \subset\left\{b_{\kappa}: \kappa=1,2, \ldots, 2 \delta_{1}\right\}$, with a condition according to our requirement of theorem that $\left|\lambda_{e, f}{ }^{\prime}\right|=3$, and removal of any vertex from $\lambda_{e, f}{ }^{\prime}$ to fulfill the definition. The result is implied in the same edge's position and contradicts our assumption with the fact that $p\left(a_{r} a_{r+1} \mid \lambda_{e, f}^{\prime}\right)=p\left(a_{s}^{\prime} a_{s+1}^{\prime} \mid \lambda_{e, f}^{\prime}\right)$, where $1 \leq r \leq 2 \delta_{3}-1$ and $1 \leq s \leq 2 \delta_{3}-4$.

Case 3: assume that $\lambda_{e, f}{ }^{\prime} \subset\left\{c_{\kappa}: \kappa=1,2, \ldots, 2 \delta_{2}-1\right\}$, with a condition according to our requirement of theorem that $\left|\lambda_{e, f}{ }^{\prime}\right|=3$, and removal of any vertex from $\lambda_{e, f}{ }^{\prime}$ to fulfill the definition. The result is implied in the same edge's position and contradicts our assumption with the fact that $p\left(b_{r+3} b_{r}^{\prime} \mid \lambda_{e, f}{ }^{\prime}\right)=p\left(a_{s} a_{s+1} \mid \lambda_{e, f}{ }^{\prime}\right)$, where $1 \leq r($ odd $) \leq 2 \delta_{1}-3$ and $1 \leq s \leq 2 \delta_{3}-1$.

Case 4: assume that $\lambda_{e, f}{ }^{\prime} \subset\left\{a_{\kappa^{\prime}}: \kappa=1,2, \ldots, 2 \delta_{3}-3\right\}$, with a condition according to our requirement of theorem that $\left|\lambda_{e, f}^{\prime}\right|=3$, and removal of any vertex from $\lambda_{e, f}{ }^{\prime}$ to fulfill the definition. The result is implied in the same edge's position and contradicts our assumption with the fact that $p\left(a_{r} a_{r+1} \mid \lambda_{e, f}{ }^{\prime}\right)=p\left(c_{s} c_{s}^{\prime} \mid \lambda_{e, f}{ }^{\prime}\right)$, where $1 \leq r \leq 2 \delta_{3}-1$ and $1 \leq s$ (odd) $\leq 2 \delta_{2}-1$.
Case 5: assume that $\lambda_{e, f}{ }^{\prime} \subset\left\{b_{\kappa^{\prime}}: \kappa=1,2, \ldots, 2 \delta_{1}-3\right\}$, with a condition according to our requirement of theorem that $\left|\lambda_{e, f}{ }^{\prime}\right|=3$, and removal of any vertex from $\lambda_{e, f}{ }^{\prime}$ to fulfill the definition. The result is implied in the same edge's position and contradicts our assumption with the fact that $p\left(a_{r} a_{r+1} \mid \lambda_{e, f}^{\prime}\right)=p\left(a_{s}^{\prime} a_{s+1}^{\prime} \mid \lambda_{e, f}^{\prime}\right)$, where $1 \leq r \leq 2 \delta_{3}-1$ and $1 \leq s \leq 2 \delta_{3}-4$

Case 6: assume that $\lambda_{e, f}{ }^{\prime} \subset\left\{c_{\kappa^{\prime}}: \kappa=1,2, \ldots, 2 \delta_{2}-1\right\}$, with a condition according to our requirement of theorem that $\left|\lambda_{e, f}{ }^{\prime}\right|=3$, and removal of any vertex from $\lambda_{e, f}{ }^{\prime}$ to fulfill the definition. The result is implied in the same edge's position and contradicts our assumption with the fact that $p\left(a_{r} a_{r+1} \mid \lambda_{e, f}^{\prime}\right)=p\left(a_{s} a_{s+1} \mid \lambda_{e, f}^{\prime}\right)$, where $1 \leq r, s \leq 2 \delta_{3}-1$.

Case 7: assume that $\lambda_{e, f}^{\prime} \subset\left\{a_{\kappa}, b_{j}: \kappa=\right.$ $\left.1,2, \ldots, 2 \delta_{3}, j=1,2, \ldots, 2 \delta_{1}\right\}$, with a condition according to our requirement of theorem that $\left|\lambda_{e, f}^{\prime}\right|=3$, and removal of any vertex from $\lambda_{e, f}^{\prime}$ to fulfill the definition. The result is implied in the same edge's position and contradicts our assumption with the fact that $p\left(b_{r} b_{r+1} \mid \lambda_{e, f}^{\prime}\right)=p\left(b_{s}^{\prime} b_{s+1}^{\prime} \mid \lambda_{e, f}^{\prime}\right)$, where $1 \leq r \leq 2 \delta_{1}-1$ and $1 \leq s \leq 2 \delta_{1}-4$.

Case 8: assume that $\lambda_{e, f}{ }^{\prime} \subset\left\{a_{\kappa}, c_{j}: \kappa=\right.$ $\left.1,2, \ldots, 2 \delta_{3}, j=1,2, \ldots, 2 \delta_{2}-1\right\}$, with a condition according to our requirement of theorem that $\left|\lambda_{e, f}^{\prime}\right|=3$, and removal of any vertex from $\lambda_{e, f}{ }^{\prime}$ to fulfill the definition. The result is implied in the same edge's position and contradicts our assumption with the fact that $p\left(b_{r+3} b_{r}^{\prime} \mid \lambda_{e, f}{ }^{\prime}\right)=p\left(b_{s}^{\prime} b_{s+1}{ }^{\prime} \mid \lambda_{e, f}{ }^{\prime}\right), \quad$ where $1 \leq r$ (odd) $\leq 2 \delta_{1}-3$ and $1 \leq s \leq 2 \delta_{1}-4$.

Case 9: assume that $\lambda_{e, f}{ }^{\prime} \subset\left\{a_{\kappa}, a_{j}^{\prime}: \kappa=1,2\right.$, $\left.\ldots, 2 \delta_{3}, j=1,2, \ldots, 2 \delta_{3}-3\right\}$, with a condition according to our requirement of theorem that $\left|\lambda_{e, f}{ }^{\prime}\right|=3$, and removal of any vertex from $\lambda_{e, f}{ }^{\prime}$ to fulfill the definition. The result is implied in the same edge's position and contradicts our assumption with the fact that $p\left(b_{r} b_{r+1} \mid \lambda_{e, f}^{\prime}\right)=p\left(b_{s+3} \mid b_{r}^{\prime} \lambda_{e, f}^{\prime}\right)$, where $1 \leq r \leq 2 \delta_{1}-1$ and $1 \leq s($ odd $) \leq 2 \delta_{1}-3$.

Case 10: assume that $\lambda_{e, f}{ }^{\prime} \subset\left\{a_{\kappa}, b_{j}^{\prime}: \kappa=\right.$ $\left.1,2, \ldots, 2 \delta_{3}, j=1,2, \ldots, 2 \delta_{1}-3\right\}$, with a condition according to our requirement of theorem that $\left|\lambda_{e, f}{ }^{\prime}\right|=3$, and removal of any vertex from $\lambda_{e, f}{ }^{\prime}$ to fulfill the definition. The result is implied in the same edge's position and contradicts our assumption with the fact that $p\left(a_{r} a_{r+1} \mid \lambda_{e, f}^{\prime}\right)=p\left(a_{s} a_{s}^{\prime} \mid \lambda_{e, f}^{\prime}\right)$, where $1 \leq r \leq 2 \delta_{3}-1$ and $1 \leq s($ odd $) \leq 2 \delta_{3}-3$.

Case 11: assume that $\lambda_{e, f}{ }^{\prime} \subset\left\{a_{\kappa}, c_{j}^{\prime}: \kappa=1,2\right.$, $\left.\ldots, 2 \delta_{3}, j=1,2, \ldots, 2 \delta_{2}-1\right\}$, with a condition 
according to our requirement of theorem that $\left|\lambda_{e, f}{ }^{\prime}\right|=3$, and removal of any vertex from $\lambda_{e, f}$ to fulfill the definition. The result is implied in the same edge's position and contradicts our assumption with the fact that $p\left(b_{r} b_{r+1} \mid \lambda_{e, f}^{\prime}\right)=p\left(b_{s}^{\prime} b_{s+1}^{\prime} \mid \lambda_{e, f}^{\prime}\right)$, where $1 \leq r \leq 2 \delta_{1}-1$ and $1 \leq s \leq 2 \delta_{1}-4$.

Case $12: \quad \quad$ assume $\quad$ that
$\lambda_{e, f}^{\prime} \subset\left\{b_{\kappa}, c_{j}: \kappa=1,2, \ldots, 2 \delta_{1}, j=1,2, \ldots, 2 \delta_{2}-1\right\}$, with a condition according to our requirement of theorem that $\left|\lambda_{e, f}{ }^{\prime}\right|=3$, and removal of any vertex from $\lambda_{e, f}{ }^{\prime}$ to fulfill the definition. The result is implied in the same edge's position and contradicts our assumption with the fact that $p\left(a_{r} a_{r+1} \mid \lambda_{e, f}^{\prime}\right)=p\left(a_{s} a_{s}^{\prime} \mid \lambda_{e, f}^{\prime}\right)$, where $1 \leq r \leq 2 \delta_{3}-1$ and $1 \leq s$ (odd) $\leq 2 \delta_{3}-3$.

Case
$\lambda_{e, f}^{\prime} \subset\left\{b_{\kappa}, a_{j}^{\prime}: \kappa=1,2, \ldots, 2 \delta_{1}, j=1,2, \ldots, 2 \delta_{3}-3\right\}$ , with a condition according to our requirement of theorem that $\left|\lambda_{e, f}{ }^{\prime}\right|=3$, and removal of any vertex from $\lambda_{e, f}{ }^{\prime}$ to fulfill the definition. The result is implied in the same edge's position and contradicts our assumption with the fact that $p\left(a_{r} a_{r+1} \mid \lambda_{e, f}^{\prime}\right)=p\left(a_{s} a_{s}^{\prime} \mid \lambda_{e, f}^{\prime}\right)$, where $1 \leq r \leq 2 \delta_{3}-1$ and $1 \leq s($ odd $) \leq 2 \delta_{3}-3$.

Case 14: assume that $\lambda_{e, f}{ }^{\prime} \subset\left\{b_{\kappa}, b_{j}^{\prime}: \kappa=1,2, \ldots, 2 \delta_{1}, j=1,2, \ldots, 2 \delta_{3}-3\right\}$, with a condition according to our requirement of theorem that $\left|\lambda_{e, f}^{\prime}\right|=3$, and removal of any vertex from $\lambda_{e, f}{ }^{\prime}$ to fulfill the definition. The result is implied in the same edge's position and contradicts our assumption with the fact that $p\left(a_{r} a_{r+1} \mid \lambda_{e, f}^{\prime}\right)=p\left(a_{s} a_{s}^{\prime} \mid \lambda_{e, f}^{\prime}\right)$, where $1 \leq r \leq 2 \delta_{3}-1$ and $1 \leq s($ odd $) \leq 2 \delta_{3}-3$.

Case 15: assume that $\lambda_{e, f}^{\prime} \subset\left\{b_{\kappa}, c_{j}^{\prime}: \kappa=1,2, \ldots, 2 \delta_{1}, j=1,2, \ldots, 2 \delta_{2}-1\right\}$, with a condition according to our requirement of theorem that $\left|\lambda_{e, f}^{\prime}\right|=3$, and removal of any vertex from $\lambda_{e, f}{ }^{\prime}$ to fulfill the definition. The result is implied in the same edge's position and contradicts our assumption with the fact that $p\left(a_{r} a_{r+1} \mid \lambda_{e, f}^{\prime}\right)=p\left(a_{s}^{\prime} a_{s+1}^{\prime} \mid \lambda_{e, f}^{\prime}\right)$, where $1 \leq r \leq 2 \delta_{3}-1$ and $1 \leq s \leq 2 \delta_{3}-4$.

Case 16: assume that $\lambda_{e, f}^{\prime} \subset\left\{c_{\kappa}, a_{j}^{\prime}: \kappa=1,2, \ldots, 2 \delta_{2}-\right.$ $\left.1, j=1,2, \ldots, 2 \delta_{3}-3\right\}$, with a condition according to our requirement of theorem that $\left|\lambda_{e, f}{ }^{\prime}\right|=3$, and removal of any vertex from $\lambda_{e, f}$, to fulfill the definition. The result is implied in the same edge's position and contradicts our assumption with the fact that $p\left(b_{r} b_{r+1} \mid \lambda_{e, f}^{\prime}\right)=p\left(b_{s}^{\prime} b_{s+1}^{\prime} \mid \lambda_{e, f}^{\prime}\right), \quad$ where $\quad 1 \leq r \leq 2 \delta_{1}-1$ and $1 \leq s \leq 2 \delta_{1}-4$.

Case 17: assume that $\lambda_{e, f}^{\prime} \subset\left\{c_{\kappa}, b_{j}^{\prime}: \kappa=1,2, \ldots, 2 \delta_{2}-1, j=1,2, \ldots, 2 \delta_{1}-\right.$ $3\}$, with a condition according to our requirement of theorem that $\left|\lambda_{e, f}^{\prime}\right|=3$, and removal of any vertex from $\lambda_{e, f}^{\prime}$ to fulfill the definition. The result is implied in the same edge's position and contradicts our assumption with the fact that $p\left(a_{r} a_{r+1} \mid \lambda_{e, f}^{\prime}\right)=p\left(a_{s}^{\prime} a_{s+1}^{\prime} \mid \lambda_{e, f}^{\prime}\right)$, where $1 \leq r \leq 2 \delta_{3}-1$ and $1 \leq s \leq 2 \delta_{3}-4$.

Case 18: assume that $\lambda_{e, f}{ }^{\prime} \subset\left\{c_{\kappa}, c_{j}^{\prime}: \kappa, j=1,2, \ldots, 2 \delta_{2}-1\right\}$, with a condition according to our requirement of theorem that $\left|\lambda_{e, f}^{\prime}\right|=3$, and removal of any vertex from $\lambda_{e, f}^{\prime}$ to fulfill the definition. The result is implied in the same edge's position and contradicts our assumption with the fact that $p\left(b_{r} b_{r+1} \mid \lambda_{e, f}^{\prime}\right)=p\left(b_{s} b_{s+1} \mid \lambda_{e, f}^{\prime}\right)$, where $1 \leq r, s \leq 2 \delta_{1}-1$.

Case 19: assume that $\lambda_{e, f}^{\prime} \subset\left\{a_{\kappa}^{\prime}, b_{j}^{\prime}: \kappa=1,2, \ldots, 2 \delta_{3}-3, j=1,2, \ldots, 2 \delta_{1}-\right.$

3 , with a condition according to our requirement of theorem that $\left|\lambda_{e, f}^{\prime}\right|=3$, and removal of any vertex from $\lambda_{e, f}^{\prime}$ to fulfill the definition. The result is implied in the same edge's position and contradicts our assumption with the fact that $p\left(b_{r} b_{r+1} \mid \lambda_{e, f}^{\prime}\right)=p\left(b_{s}^{\prime} b_{s+1}^{\prime} \mid \lambda_{e, f}^{\prime}\right)$, where $1 \leq r \leq 2 \delta_{1}-1,1 \leq s \leq 2 \delta_{1}-4$.

Case 20: assume that $\lambda_{e, f}^{\prime} \subset\left\{a_{\kappa}^{\prime}, c_{j}^{\prime}: \kappa=1,2, \ldots, 2 \delta_{3}-3, \quad j=1,2, \ldots\right.$, $\left.2 \delta_{2}-1\right\}$, with a condition according to our requirement of theorem that $\left|\lambda_{e, f}^{\prime}\right|=3$, and removal of any vertex from $\lambda_{e, f}^{\prime}$ to fulfill the definition. The result is implied in the same edge's position and contradicts our assumption with the fact that $p\left(b_{r} b_{r+1} \mid \lambda_{e, f}^{\prime}\right)=p\left(b_{s}^{\prime} b_{s+1}^{\prime} \mid \lambda_{e, f}^{\prime}\right)$, where $1 \leq r \leq 2 \delta_{1}-1$, $1 \leq s \leq 2 \delta_{1}-4$.

Case 21: assume that $\lambda_{e, f}^{\prime} \subset\left\{b_{\kappa}^{\prime}\right.$, $\left.c_{j}^{\prime}: \kappa=1,2, \ldots, 2 \delta_{1}-3, j=1,2, \ldots, 2 \delta_{2}-1\right\}$, with a condition according to our requirement of theorem that $\left|\lambda_{e, f}{ }^{\prime}\right|=3$, and removal of any vertex from $\lambda_{e, f}{ }^{\prime}$ to fulfill the definition. The result is implied in the same edge's position and contradicts our assumption with the fact that $p\left(a_{r} a_{r+1} \mid \lambda_{e, f}{ }^{\prime}\right)=p\left(a_{s}^{\prime} a_{s+1}{ }^{\prime} \mid \lambda_{e, f}^{\prime}\right)$, where $1 \leq r, s \leq 2 \delta_{1}$.

The given positions $\mathfrak{p}\left(. \mid \lambda_{e, f}\right)$ of all $5\left(\delta_{1}+\delta_{2}+\delta_{3}\right)-11$-bonds of $T\left(\delta_{1}, \delta_{2}, \delta_{3}\right)$ graph of benzenoid tripod with $\delta_{1}, \delta_{2}, \delta_{3} \geq 2$, with respect to $\lambda_{e, f}$, having $\left|\lambda_{e, f}\right|=4$, are unique and no two bonds have the same position $\mathfrak{p}$. It can also be accessed that eliminating any of arbitrary nodes from $\lambda_{e, f}$ will not affect the definition of edge resolving set. We also checked that the fault-tolerant edge resolving set $\lambda_{e, f}$ with $\left|\lambda_{e, f}\right|=3$ resulted in two edges having the same position $\mathfrak{p}$. So we can conclude that we resolve the bonds of $T\left(\delta_{1}, \delta_{2}, \delta_{3}\right)$ with four nodes. It is implied that the minimum members in the fault-tolerant edge resolving set of $T\left(\delta_{1}, \delta_{2}, \delta_{3}\right)$ are four.

Remark 2. If $T\left(\delta_{1}, \delta_{2}, \delta_{3}\right)$ is a graph of benzenoid tripod with $\delta_{1}, \delta_{2}, \delta_{3} \geq 2$, then

$$
\operatorname{dim}_{e, f}\left(T\left(\delta_{1}, \delta_{2}, \delta_{3}\right)\right)=4
$$

Proof. From the definition of fault-tolerant edge metric dimension (same as in parent concept), the concept is solemnly based on the selected subset $\left(\lambda_{e, f}\right)$ chosen in such a way that the entire edge set has unique position with respect to the selected nodes or subset. Addition or removal of any arbitrary single member of $\lambda_{e, f}$ does not affect the resolvability of edges or position of the entire edge set of graph remains unique. In Lemma 2, we already discussed the possibility of selected subset (fault-tolerant edge resolving set) and, according to the definition, its minimum possible 
cardinality. In that lemma, we choose $\lambda_{e, f}=\left\{a_{1}, b_{1}, a_{2 \delta_{3}}, b_{2 \delta_{1}}\right\}$ as a fault-tolerant edge resolving set for the graph of benzenoid tripod or $T\left(\delta_{1}, \delta_{2}, \delta_{3}\right)$ for all the possible combinatorial values of $\delta_{1}, \delta_{2}, \delta_{3} \geq 2$. We also proved in such lemma that $\left|\lambda_{e, f}\right|=4$ is the least possible cardinality of fault-tolerant edge resolving set for the benzenoid tripod $T\left(\delta_{1}, \delta_{2}, \delta_{3}\right)$. It is enough for the proof of what we claim in the statement that fault-tolerant edge metric dimension of benzenoid tripod is four, which completes the proof.

\section{Conclusion}

Mathematical chemistry, particularly graphical chemistry, has made it simpler to examine complicated networks and chemical structures in their simplest forms. Similarly, resolvability is a parameter in which the complete node or edge set, and occasionally both, reconfigure themselves into unique outfits in order to call or access them. Edge metric dimension is also a parameter with this property to gain each edge into a unique form. In this work, we consider benzenoid tripod structure to achieve its resolvability and found its minimum edge-resolving set. We concluded that edge metric and fault-tolerant edge metric resolving set are with constant and exact number of members for this structure.

\section{Data Availability}

There are no data associated with this manuscript.

\section{Conflicts of Interest}

The authors declare that they have no conflicts of interest.

\section{References}

[1] B. Yang, M. Rafiullah, H. M. A. Siddiqui, and S. Ahmad, "On resolvability parameters of some wheel-related graphs," Journal of Chemistry, vol. 2019, Article ID 9259032, 9 pages, 2019.

[2] Y. Zhang and S. Gao, "On the edge metric dimension of convex polytopes and its related graphs," Journal of Combinatorial Optimization, vol. 39, no. 2, pp. 334-350, 2020.

[3] M. Ahsan, Z. Zahid, S. Zafar, A. Rafiq, M. S. Sindhu, and M. Umar, "Computing the edge metric dimension of convex polytopes related graphs," The Journal of Mathematics and Computer Science, vol. 22, no. 2, pp. 174-188, 2020.

[4] Z. Raza and M. S. Bataineh, "The comparative analysis of metric and edge metric dimension of some subdivisions of the wheel graph," Asian-European Journal of Mathematics, vol. 14, no. 4, Article ID 2150062, 2020.

[5] I. G. Yero, "Vertices, edges, distances and metric dimension in graphs," Electronic Notes in Discrete Mathematics, vol. 55, pp. 191-194, 2016.

[6] J. B. Liu, Z. Zahid, R. Nasir, and W. Nazeer, "Edge version of metric dimension anddoubly resolving sets of the necklace graph," Mathematics, vol. 6, no. 43, 2018.

[7] M. Azeem and M. F. Nadeem, "Metric-based resolvability of polycyclic aromatic hydrocarbons," European Physical Journal Plus, vol. 136, no. 395, 2021.
[8] M. N. A. T. Iqbal and S. A. H. Bokhary, "The k-size edge metric dimension of graphs," Journal of Mathematics, vol. 2020, Article ID 1023175, 7 pages, 2020.

[9] M. Wei, J. Yue, J. Yue, and X. Zhu, "On the edge metric dimension of graphs," AIMS Mathematics, vol. 5, no. 5, pp. 4459-4465, 2020.

[10] M. Knor, S. Majstorović, A. T. Masa Toshi, R. Škrekovski, and I. G. Yero, "Graphs with the edge metric dimension smaller than the metric dimension," Applied Mathematics and Computation, vol. 401, Article ID 126076, 2021.

[11] V. Filipovic, A. Kartelj, and J. Kratica, "Edge metric dimension of some generalized peterson graphs," Results in Mathematics, vol. 74182 pages, 2019.

[12] M. S. Bataineh, N. Siddiqui, and Z. Raza, "Edge metric dimension of \$k\$ multiwheel graph," Rocky Mountain Journal of Mathematics, vol. 50, no. 4, pp. 1175-1180, 2020.

[13] P. Slater, "'Leaves of trees," proceeding of the 6th southeastern conference on combinatorics, graph theory, and computing," Congressus Numerantium, vol. 14, pp. 549-559, 1975.

[14] F. Harary and R. A. Melter, "On the metric dimension of a graph,” Ars Combinatoria, vol. 2, pp. 191-195, 1976.

[15] G. Chartrand, E. Salehi, and P. Zhang, "The partition dimension of a graph," Aequationes Mathematicae, vol. 59, no. 1, pp. 45-54, 2000.

[16] G. Chartrand, L. Eroh, M. A. Johnson, and O. R. Oellermann, "Resolvability in graphs and the metric dimension of a graph," Discrete Applied Mathematics, vol. 105, no. 1-3, pp. 99-113, 2000.

[17] M. Johnson, "Structure-activity maps for visualizing the graph variables arising in drug design," Journal of Biopharmaceutical Statistics, vol. 3, no. 2, pp. 203-236, 1993.

[18] S. Khuller, B. Raghavachari, and A. Rosenfeld, "Landmarks in graphs," Discrete Applied Mathematics, vol. 70, no. 3, pp. 217-229, 1996.

[19] S. Söderberg and H. S. Shapiro, "A combinatory detection problem," The American Mathematical Monthly, vol. 70, no. 10, pp. 1066-1070, 1963.

[20] P. Manuel, R. Bharati, I. Rajasingh, and C. Monica M, "On minimum metric dimension of honeycomb networks," Journal of Discrete Algorithms, vol. 6, no. 1, pp. 20-27, 2008.

[21] A. Sebö and E. Tannier, "On metric generators of graphs," Mathematics and Operational Research, vol. 29, pp. 383-393, 2004.

[22] M. Perc, J. Gómez-Gardeñes, A. Szolnoki, L. M. Floría, and Y. Moreno, "Evolutionary dynamics of group interactions on structured populations: a review," Journal of The Royal Society Interface, vol. 10, no. 80, Article ID 20120997, 2013.

[23] M. Perc and A. Szolnoki, "Coevolutionary games-a mini review," Biosystems, vol. 99, no. 2, pp. 109-125, 2010.

[24] Z. Hussain, M. Munir, M. Choudhary, and S. M. Kang, "Computing metric dimension and metric basis of $2 \mathrm{~d}$ lattice of alpha-boron nanotubes," Symmetry, vol. 10, 2018.

[25] S. Krishnan and B. Rajan, "Fault-tolerant resolvability of certain crystal structures," Applied Mathematics, vol. 7, no. 7, pp. 599-604, 2016.

[26] H. M. A. Siddiqui and M. Imran, "Computing metric and partition dimension of 2-dimensional lattices of certain nanotubes," Journal of Computational and Theoretical Nanoscience, vol. 11, no. 12, pp. 2419-2423, 2014.

[27] M. Hauptmann, R. Schmied, and C. Viehmann, "Approximation complexity of metric dimension problem," Journal of Discrete Algorithms, vol. 14, pp. 214-222, 2012.

[28] H. R. Lewis, M. Garey, and D. Johnson, "Michael R. ПGarey and David S. Johnson. Computers and intractability. A guide 
to the theory of NP-completeness. W. H. Freeman and Company, San Francisco1979, x +338 pp," The Journal of Symbolic Logic, vol. 48, no. 2, pp. 498-500, 1983.

[29] M. A. Johnson, "Browsable structure-activity datasets," Advances in Molecular Similarity, pp. 153-170, JAI Press Connecticut, Stamford, CT, USA, 1998.

[30] A. Kelenc, N. Tratnik, and I. G. Yero, "Uniquely identifying the edges of a graph: the edge metric dimension," Discrete Applied Mathematics, vol. 251, pp. 204-220, 2018.

[31] M. F. Nadeem, M. Azeem, and A. Khalil, "The locating number of hexagonal möbius ladder network," Journal of Applied Mathematics and Computing, vol. 66, no. 1-2, pp. 149-165, 2020.

[32] B. Deng, M. F. Nadeem, and M. Azeem, "On the edge metric dimension of different families of möbius networks," Mathematical Problems in Engineering, vol. 20219 pages, Article ID 6623208, 2021.

[33] A. Ali, W. Nazeer, M. Munir, and S. M. Kang, "M-polynomials and topological indices of zigzagand rhombic benzenoid systems," Open Chemistry, vol. 16, no. 73-78, pp. 122-135, 2018.

[34] C. P. Chou, Y. Li, and H. A. Witek, "Zhang-zhang polynomials of various classes of benzenoid systems," MATCH Communications in Mathematicaland in Computer Chemistry, vol. 68, pp. 31-64, 2012.

[35] M. K. Jamil, M. Imran, and K. A. Sattar, "Novel face index for benzenoid hydrocarbons," Mathematics, vol. 8, no. 312, 2020.

[36] A. N. A. Koam, A. Ahmad, M. E. Abdelhag, and M. Azeem, "Metric and fault-tolerant metric dimension of hollow coronoid," IEEE Access, vol. 9, pp. 81527-81534, 2021. 